\title{
Comparison of Gene Expression in Breast Cancer Using Laser Capture Microdissection and Quantitative RT-PCR
}

\author{
Xiaojuan Wang ${ }^{1}$, Misa Nakamura ${ }^{1}$, Ichiro Mori' ${ }^{1}$, Koichi Takeda ${ }^{1}$, Takaomi Suzuma ${ }^{1,2}$, \\ Goro Yoshimura ${ }^{1,2}$, Takeo Sakurai ${ }^{1,2}$ and Kennichi Kakudo ${ }^{1}$ \\ ${ }^{1}$ Department of Pathology, Wakayama Medical University, 811-1 Kimiidera, Wakayama City, Wakayama 641-8509, Japan and \\ ${ }^{2}$ Affiliated Kihoku Hospital, Wakayama Medical University, Japan
}

Received May 26, 2003; accepted August 13, 2003

\begin{abstract}
Amplification and deletion are the most common mechanisms leading genes to gene deregulation in breast cancer, but the relevance, the role and timing of most of the described genetic abnormalities are still unclear. The significance and usefulness of those genetic abnormalities will have to be established by the use of reliable and reproducible methodologies. In this study, we used the advanced technique of laser capture microdissection to obtain pure tumor cell populations and normal epithelium in paired breast tissue samples from the
\end{abstract}

same patient, and submitted them to further analysis by real-time quantitative RT-PCR for a rapid, objective and reliable quantification of even small abnormalities. We will also introduce and recommend a simple staining method and a suitable thickness of section for LCM and quality RNA extraction from the tissue samples. We believe that this method will provide more detailed evidence of gene expression in the study of cancers, especially in multi-step cancer carcinogenesis.

Key words: laser capture microdissection, real-time RT-PCR, cancer, calcitonin receptor

In breast cancer, amplification and deletion are the most common mechanisms leading genes to gene deregulation [2]. Several gene abnormalities have been reported to be involved $[1,9,10,17,18]$, but the number and identity of the abnormal genes relevant to mammary carcinogenesis are unknown. Despite abundant data, the relevance, the roles and timing of most of the described genetic abnormalities in breast cancer have yet to be clarified. The significance and usefulness of these findings will have to be established using reliable and reproducible methodologies. Furthermore, in the study of multi-step breast carcinogenesis, contamination from bystander cells may distort amplification or deletion data. This distortion by contaminating bystander cells is especially important in the study of small, precancerous lesions that typically show lower levels of gene amplifica-

Correspondence to: Kennichi Kakudo, Department of Pathology, Wakayama Medical University, 811-1 Kimiidera, Wakayama City, Wakayama 641-8509, Japan. tion and thus fail to be detected.

In this study, we used the advanced technique of laser capture microdissection (LCM) to obtain pure tumor cell populations and normal epithelium in paired breast tissue samples from the same patient, and submitted them to further analysis by real-time quantitative RT-PCR for rapid, objective and reliable quantification of even small abnormalities. We also introduced and recommended a simple staining method and a suitable thickness of section for this LCM and quality RNA extraction from tissue sample.

\section{Tissues and preparation}

Samples of breast tissue and primary breast cancer were obtained from patients treated at the Affiliated Kihoku Hospital, Wakayama Medical University, Japan. Informed consent was obtained from each subject and the study was approved by the Institutional Review Board of Wakayama Medical University. The samples were obtained immediately after resection and cut serially to identify the main tumor 
and normal breast tissue. The tissue was embedded in Tissue-tek OCT (Miles Inc. Diagnostics Division, Elkhart, $\mathrm{IN}$ ) and stored at $-70^{\circ} \mathrm{C}$ until further analysis.

\section{LCM and RNA extraction}

Frozen sections of $16 \mu \mathrm{m}$ thickness were prepared from normal and cancer tissue blocks. One set of slides were stained with hematoxylin and eosin and examined under a microscope by two pathologists to provide a detailed confirmation of the identification of the cancer and the normal breast tissues. Frozen sections were then mounted on foilslides (Matsunami Glass Ltd. Osaka, Japan) and stained with toluidine blue to identify cancer cells against normal breast epithelial cells or stroma (Fig. 1a-d).

Microdissection of target cells was performed using the AS-LCM System (Leica Microsystems, Germany), according to the protocol provided by the manufacturer. Total RNA was isolated from harvested cells using TRIzol (Sigma, St. Louis, MO, USA), according to the manufacturer's protocol. Total RNA was dissolved in $10 \mu$ of diethyl pyrocarbonate-treated $\mathrm{H}_{2} \mathrm{O}$. All RNAs were verified for integrity by observing RT-PCR products for $\beta$-actin before using them for quantitative RT-PCR.

\section{Real-time RT-PCR assay}

Total RNA from the paired samples were analyzed using one-step RT-PCR, using the TaqMan ABI 7000 sequence detection system (PE Applied Biosystems, Foster, CA, USA), according to TaqMan protocol. Each sample was used for RT-PCR to amplify target and a housekeeping gene, GAPDH, which served as an endogenous standard to correct them for losses during the sample preparation and for the different cell numbers in samples. RT-PCR was performed to amplify GAPDH in selected cases showing similarly amplified levels in the samples. The primer sequences for amplification were described as follows: for GAPDH, forward primer 5'-GAA GGT GAA GGT CGG AGT C-3', reverse primer 5'-GAA GAT GGT GAT GGG ATT C-3' and probe 5'-(JOE)-CA AGC TTC CCG TTC TCA GCC-(TAMRA)3'. The primers and probe for CTR were purchased from Applied Biosystems Japan Ltd. (Applied Biosystems Japan Ltd., Tokyo, Japan). One step real-time RT-PCR was carried
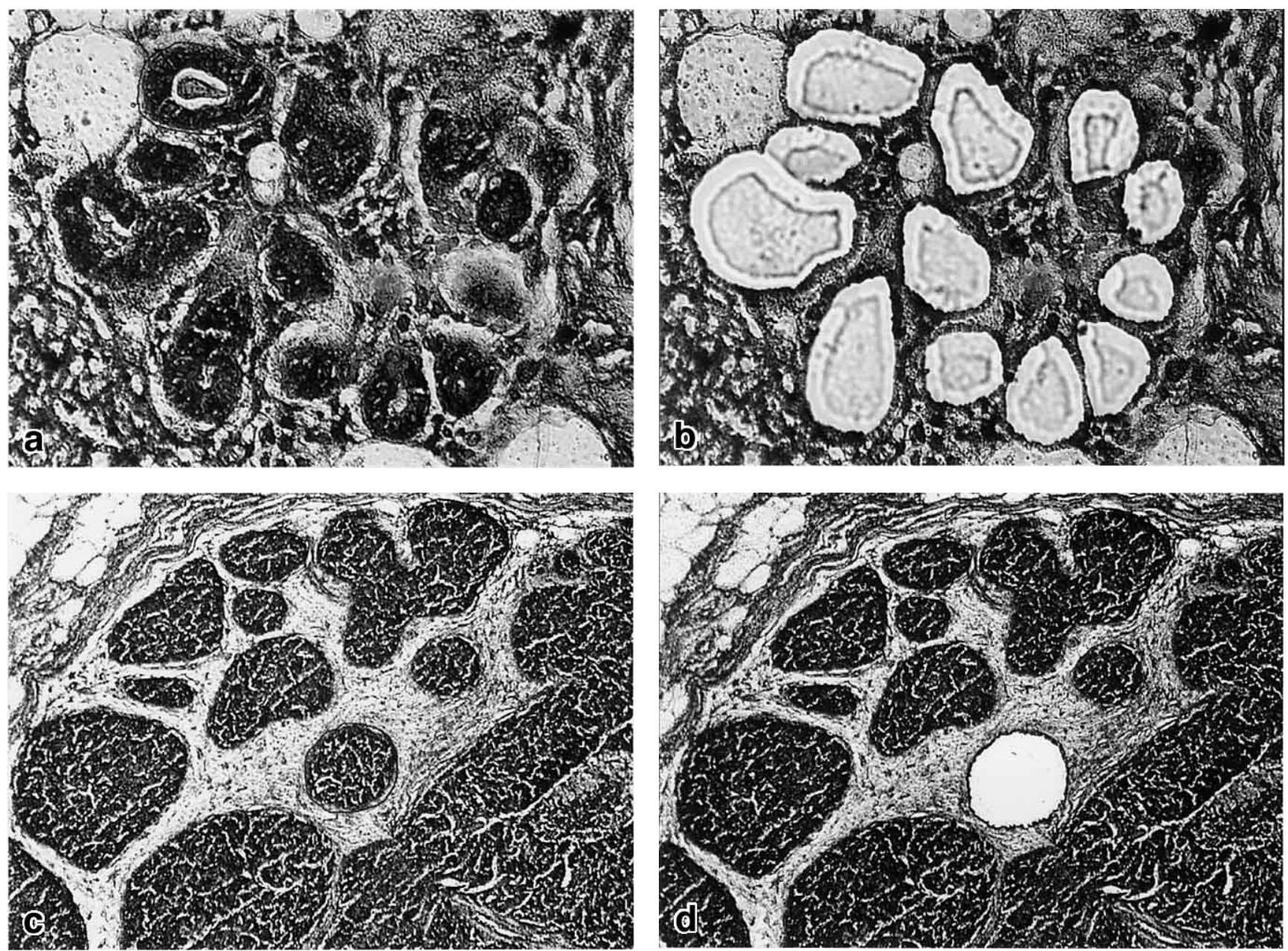

Fig. 1. Paired samples were collected using laser capture microdissection. (a-b) normal breast and (c-d) breast cancer before and after microdissection, respectively. (Stained by Toluidine Blue, $\times 100$ ) 
out using TaqMan EZ RT-PCR reagents (Applied Biosystems, NJ, USA). RNA was reverse-transcribed to cDNA and amplified using the PCR process in a single tube and single enzyme system, without subsequent addition of enzymes or buffers. Each RT-PCR reaction contained the following: $1 \times$ TaqMan buffer, $3 \mathrm{mM}$ manganese acetate, $300 \mu \mathrm{M}$ each of dATP, dCTP and dGTP, $600 \mu \mathrm{M}$ dUTP, $200 \mu \mathrm{M}$ primers, $100 \mu \mathrm{M}$ probe, $\mathrm{rTth}$ DNA polymerase $0.1 \mathrm{U} / \mu \mathrm{l}$, AmpErase UNG (uracil N-glycosylase) $0.01 \mathrm{U} / \mu \mathrm{l}$ and $1 \mu \mathrm{l}$ total RNA for template in $50 \mu \mathrm{l}$ of reaction volume, which was extracted from laser-captured cells containing an unknown amount of RNA.

The reaction conditions were as follows: after initial 2 min incubation at $50^{\circ} \mathrm{C}$ for UNG activity to prevent carryover reactions, RT was carried out for $30 \mathrm{~min}$ at $60^{\circ} \mathrm{C}$ and the reaction was terminated by heating at $95^{\circ} \mathrm{C}$ for $10 \mathrm{~min}$. Then 50 amplification cycles were performed at $94^{\circ} \mathrm{C}$ for $20 \mathrm{sec}$ and $60^{\circ} \mathrm{C}$ for $1 \mathrm{~min}$. PCR reactions were performed in 96-well optical reaction plates (Applied Biosystems). All experiments were performed in triplicate. A no-template control was used in every run to exclude false-positive results. The TaqMan probe consisted of an oligonucleotide with a 5'-reporter dye and a 3'-quencher dye. A fluorescent reporter dye, FAM (6-carboxyfluorenscein), was covalently linked to the 5 ' end of the oligonucleotide of CTR. JOE (6-carboxy-4,5-dichloro-2,7-di-menthoxyfluorescein) was linked to the GAPDH as an internal control.

The initial template concentration was derived from the cycle number at which the fluorescent signal crossed a threshold in the exponential phase of the PCR. The $\mathrm{Ct}$ value was taken as the time point during PCR at which the signal is generated due to probe hydrolysis crossing this threshold. The number of PCR cycles required to reach the threshold (Ct) signal was inversely proportional to the number of copies of the target sequence in each sample. In each sample, the real-time quantification of target transcript was determined based on the threshold cycles of the target and the internal reference gene. The results were also plotted as average target $\mathrm{Ct}$ values for each triplicate sample minus the average triplicate values for GAPDH. Differences between the tumor and normal samples were calculated using the formula $2 \exp \left(\mathrm{Ct}_{\text {tumor }}-\mathrm{Ct}_{\text {normal }}\right)$ and expressed as a fold change in expression $[13,15,20]$.

\section{RT-PCR assay}

To further confirm the target gene expression, the SuperScript ${ }^{\mathrm{TM}}$ One-step RT-PCR with Platinum Taq (Invitrogen Life Technologies Co., CA) was used to synthesize cDNA and PCR, as described by the manufacturer. PCR reactions were performed using one cycle at $55^{\circ} \mathrm{C}$ for $30 \mathrm{~min}$ for cDNA synthesis and at $94^{\circ} \mathrm{C}$ for $2 \mathrm{~min}$ for pre-denaturation, followed by 45 cycles of $94^{\circ} \mathrm{C}$ for $30 \mathrm{sec}$ for denaturation, $55^{\circ} \mathrm{C}$ for $30 \mathrm{sec}$ for annealing and $72^{\circ} \mathrm{C}$ for $30 \mathrm{sec}$ for extension, followed by $7 \mathrm{~min}$ at $72^{\circ} \mathrm{C}$ for final extension (Perkin Elmer, Gene Amp PCR System 9700, Norwalk CT, USA). The products were electrophoresed in $3 \%$ agarose gels and stained with ethidium bromide.

\section{Discussion}

In this study, we quantitatively analyzed CTR gene expression in paired breast samples. Of selected examples of three cases (Fig. 2), one case demonstrated CTR expression decreased in cancer cells when compared with normal epithelium, whereas an increased expression was found in one cancer, while no significant change was seen in another cancer. RT-PCR further confirmed that both paired tissues expressed CTR mRNA. These results not only demonstrated that the target gene was expressed in both normal tissue and breast cancer, but also revealed the different expression between normal epithelium and cancer cells. This kind of comparison offers evidence of varied target gene expression during the development and progression of breast cancers, and may help us accurately detect and identify those genes which are involved in carcinogenesis and the progression of breast cancer.

Alteration in gene expression is one of the most important mechanisms leading to a neoplastic transformation. An exact quantitative analysis of this phenomenon during different stages of tumor development will contribute to our understanding of tumor evolution and progression. Investigation of the pathogenic or prognostic significance of gene expression requires a reliable, sensitive and objective method, especially in limited small lesions. Up to now, numerous studies have reported expression abnormalities of a great number of genes involved in carcinogenesis of mammary gland but few have demonstrated an accurate distinction between the normal and the cancer, or between varied gene expressions from the normal, ductal hyperplasia, atypical ductal hyperplasia, carcinoma in situ, to invasive cancer and even metastatic cancer. This may be partly because of poor resolution of in situ hybridization methods as well as the unsuitablility of immunohistochemical analysis for quantitative study.

Laser-assisted microdissection allows us to obtain pure groups of cells microscopically [19] and to circumvent the problem of tissue heterogeneity and contamination from bystander cells or tissues. Under microscopic control, an exact separation of target cell complexes from surrounding stroma cells and contact-free isolation of the cells is possible. There are currently three commercially available systems designed specifically for LCM: PixCell by Arcturus (Mountain View, CA), PALM by P.A.L.M. Mikrolaser Technologie (Wolfratshausen, Germany) and the Leica AS LMD by Leica (Heidelberg, Germany).

The Leica AS-LMD, introduced as the third generation of laser microdissection systems, uses a pulsed UV laser similar to the PALM on an upright microscope. The laser bean may be moved with a software-controlled mirror system to select cells to isolate them or to delineate an area to be dissected. The dissected material is allowed to fall by gravity into a cap and may thereafter be used for isolating proteins or genetic material. Because Leica AS-LMD uses a laser to ablate a contour line around a single cell, the width of the cut achieved is much smaller than other systems, with a mini- 

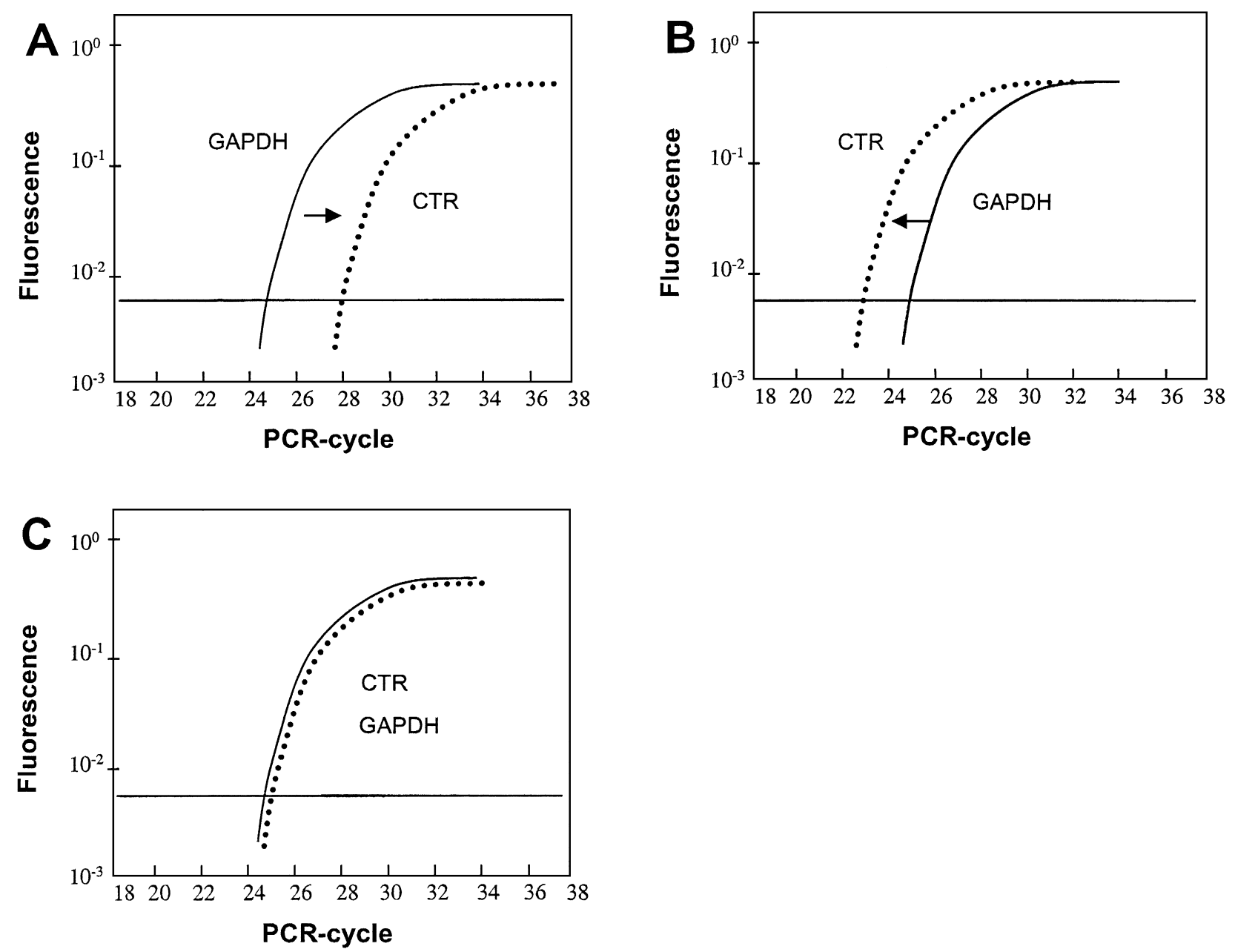

Fig. 2. Illustration of real-time quantitative RT-PCR in selected three cancers. Case A (53-year-old, invasive ductal carcinoma): A decreased gene expression causes a lower $\mathrm{Ct}$ value, shifting the amplification plot to the right. Case B (44-year-old, invasive ductal carcinoma): An increased gene expression causes a higher $\mathrm{Ct}$ value, shifting the amplification plot to the left. Case $\mathrm{C}$ (53-year-old, invasive ductal carcinoma): No change of gene expression shows similar $\mathrm{Ct}$ value to GAPDH.

mum cut size of less then $2.5 \mu \mathrm{m}$. Furthermore, the Leica system relies largely on the ablation of the polyethylene naphthalate foil for dissection, offering more freedom for the preparation of the specimen, such that the tissue sections may be much thicker with various fixations available. By contrast, Arcturus system requires strict the thickness of the section, method of fixation of the tissue and staining. Humidity between slide and membrane also do not seem to hamper proper membrane cutting in the Leica system, as compared to the PALM and Arcturus systems.

In this study, the total RNA was harvested from a sample of less than 1000 cells. We compared serial sections at thicknesses of $5 \mu \mathrm{m}, 10 \mu \mathrm{m}, 16 \mu \mathrm{m}$ and $20 \mu \mathrm{m}$ for microdissection. The $5 \mu \mathrm{m}$ and $10 \mu \mathrm{m}$ thick sections were easily cut by laser beam but much more cells were needed for definite RNA extractions. The $20 \mu \mathrm{m}$ thick sections were too thick to be cut easily because of amount of fibrous tissue in the stroma. The $16 \mu \mathrm{m}$ thick sections yielded the best results in terms of reliability and definition of the subsequent RT-
PCR in this study. We also experienced hematoxylin and eosin (HE) and toluidine blue staining for microdissection. Compared to HE staining, toluidine blue staining, which was also recommended by Lawrie [12] and Craven [4], is simple and requires fewer steps, thus resulting in less RNA loss and needing fewer cells for definite RNA extraction.

The novel real-time RT-PCR technology, which utilizes the 5'exonuclease activity of the Tag polymerase in combination with a fluorescent hybridization probe indicating PCR product accumulation, offers the advantage of a very sensitive and exact quantification of a minute amount of nucleic acids $[3,8,14]$. The most reliable method previously established for the detection of gene expression was Northern blotting $[11,21]$. The big disadvantage is the requirement of a relatively large amount of fresh sample to obtain sufficient RNA. This excludes the analysis of small lesions or small amounts of sample. In addition, correlation of gene expression with morphology is not possible and the sensitivity is limited because of contamination by non- 
purposed cells. In contrast to Northern blotting, conventional quantitative RT-PCR requires less material and is a powerful and highly reproducible technique for the quantitative analysis of gene expression $[6,7]$.

Fluorescence in situ hybridization is a quantitative molecular analysis combining tissue morphology and is already widely used for the detection of gene amplification or expression $[5,16]$. But small lesions, such as atypical ductal hyperplasia and ductal carcinoma in situ, are difficult to study using fluorescence in situ hybridization, because of the often poorly preserved morphological details after hybridization and the technical difficulties with reproducible quantification of hybridization signal, especially low-level amplifications. The biggest limitation of fluorescence in situ hybridization technique is the very restricted availability of standardized probes. As real-time PCR technology does not need long hybridization probes, the flexibility and versatility of the primers and probe offer a clear advantage especially in areas of research which should not be restricted by the availability of the hybridization probes.

In this study we demonstrated that the combination of LCM and real-time RT-PCR allows for the quantitative assessment of gene alteration in microscopically selected pure cells in small amounts of sample. We believe that this method in combination with immunohistochemistry and in situ hybridization analysis will provide more detailed evidence of gene controls and change of products.

\section{Acknowledgments}

We wish to dedicate this paper to the late Professor Keiichi Watanabe, School of Medicine, Tokai Universtiy for his helpful advice in conducting this study.

This study was supported by a Grant-in-Aid for Scientific Research (No. 10045073) from the Ministry of Education of the Japan-China Medical Association, Scientific Research C (No. 14570155) and foreigner's research grant from the Wakayama Foundation for the Promotion of Medicine.

\section{References}

1. Abou-Elella, A., Gramlich, T., Fritsch, C. and Gansler, T. (1996) c-myc amplification in hepatocellular carcinoma predicts unfavorable prognosis. Mod. Pathol. 9; 95-98.

2. Bergstein, I. (1999) Molecular alterations in breast cancer. In "Breast Cancer: Molecular Genetics, Pathogenesis, and Therapeutics", ed. by A. M. Bowcock, Humana Press, Totowa, pp. 143-170.

3. Bieche, I., Olivi, M., Champeme, M. H., Vidaud, D., Lidereau, R. and Vidaud, M. (1998) Novel approach to quantitative polymerase chain reaction using real time detection: application to the detection of gene amplification in breast cancer. Int. J. Cancer
78; 661-666.

4. Craven, R. A., Totty, N., Harnden, P., Selby, P. J. and Banks, R. E. (2002) Laser capture microdissection and two-dimensional polyacrylamide gel electrophoresis: evaluation of tissue preparation and sample limitations. Am. J. Pathol. 160; 815-822.

5. Fletcher, J. A. (1999) DNA in situ hybridization as an adjunct in tumor diagnosis. Am. J. Clin. Pathol. 112; S11-S18.

6. Gibson, U. E., Heid, C. A. and Williams, P. M. (1996) A novel method for real time quantitative RT-PCR. Genome Res. 6; 9951001.

7. Heid, C. A., Stevens, J., Livad, K. J. and Williams, P. M. (1996) Real time quantitative PCR. Genome Res. 6; 986-994.

8. Holland, P. M., Abramson, R. D., Watson, R. and Gelfand, D. H. (1991) Detection of specific polymerase chain reaction product by utilizing the 5'-3' exonuclease activity of Thermus aquaticus DNA polymerase. Proc. Natl. Acad. Sci. U S A 88; 7276-7280.

9. Knuutila, S., Bjorkqvist, A. M., Autio, K., Tarkkanen, M., Wolf, M., Monni, O., Szymanska, J., Larramendy, M. L., Tapper, J., Pere, H., EI-Rifai, W., Hemmer, S., Wasenius, V. M., Vidgren, V. and Zhu, Y. (1998) DNA copy number amplifications in human neoplasms: review of comparative genomic hybridization studies. Am. J. Pathol. 152; 1107-1123.

10. Kreipe, H., Feist, H., Fischer, L., Felgner, J., Heidorn, K., Mettler, L. and Parwaresch, R. (1993) Amplification of c-myc but not of $c-e r b B-2$ is associated with high proliferative capacity in breast cancer. Cancer Res. 53; 1956-1961.

11. Lackhart, D. J. and Winzeler, E. A. (2000) Genomics, gene expression and DNA arrays. Nature 405; 827-836.

12. Lawrie, L. C., Curran, S., McLeod, H. L., Fothergill, J. E. and Murray, G. I. (2001) Application of laser capture microdissection and proteomics in colon cancer. Mol. Pathol. 54; 253-258.

13. Lehmann, U., Langer, F., Feist, H., Glockner, S., Hasemeier, B. and Kreipe, H. (2002) Quantitative assessment of promoter hypermethylation during breast cancer development. Am. J. Pathol. $160 ; 605-612$.

14. Livak, K. J., Flood, S. J., Marmaro, J., Giusti, W. and Deetz, K. (1995) Oligonucleotides with fluorescent dyes at opposite ends provide a quenched probe system useful for detecting PCR product and nucleic acid hybridization. PCR Methods Appl. 4; 357-362.

15. Pfaffl, M. W. (2001) A new mathematical model for relative quantification in real-time RT-PCR. Nucleic Acids Res. 29; e45.

16. Pinkel, D., Straume, T. and Gray, J. W. (1986) Cytogenetic analysis using quantitative, high-sensitivity, fluorescence hybridization. Proc. Natl. Acad. Sci. U S A 83; 2934-2938.

17. Schwab, M. (1993) Amplification of N-mys as a prognostic marker for patients with neuroblastoma. Semin. Cancer Biol. 4; 13-18.

18. Schwab, M. (1998) Amplification of oncegenes in human cancer cells. Bioessays 20; 473-479.

19. Simone, N. L., Bonner, R. F., Gillespie, J. W., Emmert-Buck. M. R. and Liotta, L. A. (1998) Laser-capture microdissection: opening the microscopic frontier to molecular analysis. Trends Genet. $14 ; 272-276$.

20. Xu, L. L, Stackhouse, B. G., Florence, K., Zhang, W., Shanmugam, N., Sesterhenn, I. A., Zou, Z., Srikantan, V., Augustus, M., Roschke, V., Carter, K., McLeod, D. G., Moul, J. W., Soppett, D. and Srivastava, S. (2000) PSGR, a novel prostatespecific gene with homology to a $G$ protein-coupled receptor, is overexpressed in prostate cancer. Cancer Res. 60; 6568-6572.

21. Wahl, G. M., Meinkoth, J. L. and Kimmel, A. R. (1987) Northern and Southern blots. Methods Enzymol. 152; 572-581. 